ARTICLE

DOI: $10.1038 / \mathrm{s} 41467-017-00049-x$

\title{
A novel humanized mouse lacking murine P450 oxidoreductase for studying human drug metabolism
}

Mercedes Barzi ${ }^{1}$, Francis P. Pankowicz ${ }^{1,2}$, Barry Zorman ${ }^{3}$, Xing Liu4 ${ }^{4}$, Xavier Legras ${ }^{1}$, Diane Yang ${ }^{1,2}$,
Malgorzata Borowiak, $2,5,6,7,8$, Beatrice Bissig-Choisat1, ${ }^{1,5}$ Pavel Sumazin ${ }^{3,7}$, Feng Li ${ }^{4,5}$ \& Karl-Dimiter Bissig, 2,5,6,7

Only one out of 10 drugs in development passes clinical trials. Many fail because experimental animal models poorly predict human xenobiotic metabolism. Human liver chimeric mice are a step forward in this regard, as the human hepatocytes in chimeric livers generate human metabolites, but the remaining murine hepatocytes contain an expanded set of P450 cytochromes that form the major class of drug-metabolizing enzymes. We therefore generated a conditional knock-out of the NADPH-P450 oxidoreductase (Por) gene combined with $1 / 2 \mathrm{rg}^{-/-} / \mathrm{Rag}^{-/ /} / \mathrm{Fah}^{-/-}$(PIRF) mice. Here we show that homozygous PIRF mouse livers are readily repopulated with human hepatocytes, and when the murine Por gene is deleted $(<5 \%)$, they predominantly use human cytochrome metabolism. When given the anticancer drug gefitinib or the retroviral drug atazanavir, the Por-deleted humanized PIRF mice develop higher levels of the major human metabolites than current models. Humanized, murine Por-deficient PIRF mice can thus predict human drug metabolism and should be useful for preclinical drug development.

\footnotetext{
${ }^{1}$ Center for Cell and Gene Therapy, Stem Cells and Regenerative Medicine Center, Baylor College of Medicine, Houston TX-77030, USA. ${ }^{2}$ Graduate Program, Department of Molecular and Cellular Biology, Baylor College of Medicine, Houston TX-77030, USA. ${ }^{3}$ Texas Children's Cancer Center, Department of Pediatrics, Baylor College of Medicine, Houston TX-77030, USA. ${ }^{4}$ Alkek Center for Molecular Discovery, Advanced Technology Core, Baylor College of Medicine, Houston TX-77030, USA. ${ }^{5}$ Department of Molecular and Cellular Biology, Baylor College of Medicine, Houston TX-77030, USA. ${ }^{6}$ Program in Developmental Biology, Baylor College of Medicine, Houston TX-77030, USA. ${ }^{7}$ Dan L. Duncan Cancer Center, Baylor College of Medicine, Houston TX-77030, USA. ${ }^{8}$ McNair Medical Institute, Houston TX-77030, USA. Correspondence and requests for materials should be addressed to K.-D.B. (email: bissig@bcm.edu)
} 
$\mathrm{H}$ epatotoxicity and cutaneous hypersensitivity reactions are the most common reasons for toxicity-related termination of drugs in human clinical trials ${ }^{1}$, and they are also the most poorly predicted by results in animal studies. The difficulty is that drug metabolism depends on NADPH-P450 cytochromes, but whereas human cytochrome clusters contain 27 putatively functional genes, the mouse cytochrome clusters contain nearly three times as many ${ }^{2}$. More to the point, the functions of many murine and human cytochromes have yet to be elucidated, and the interspecies matches of known functions are not particularly $\operatorname{good}^{3}$. This makes accurate prediction of human drug metabolism in the mouse quite challenging.

Since the primary site of drug metabolism is the liver, human liver chimeric mice are an attractive alternative to conventional animal models. Isolated human hepatocyte can repopulate the murine liver of several mouse models ${ }^{4-11}$ and several groups have used human liver chimeric mice for evaluation of drug toxicity ${ }^{12-16}$ and identification of human metabolites ${ }^{17-19}$. The shortcoming of these chimeric mice is the remaining murine liver tissue. We have previously shown that human liver chimerism averages $42 \%$ in the $\mathrm{Fah}^{-/-} / \mathrm{Rag}^{-/-} / \mathrm{Il}^{2} \mathrm{rg}^{-/-}$(FRG) strain and maximal human chimerism $(\sim 95 \%)$ is reached in $<5 \%$ of animals ${ }^{20}$. Moreover, high chimerism on the cellular level does not take into account different expression levels and affinities of murine drug metabolizing enzymes. Only a complete liver humanization would allow human drug metabolism, but considering the many interspecies incompatibilities of ligands and receptors this might be a very challenging task. An alternative approach would be to inhibit drug metabolism in the remaining murine liver. Indeed, recently the urokinase-type plasminogen activator/severe combined immunodeficiency (uPA/SCID) mouse ${ }^{21}$ have been crossed with the cytochrome $3 \mathrm{a}(\mathrm{Cyp} 3 a)$ knock-out mouse, generating a strain which can be repopulated with human hepatocytes and is deficient of one of the main human drug metabolizing cytochrome clusters $^{22,23}$. Unfortunately, these mice massively upregulate several other cytochrome clusters, which defeats the purpose of the model ${ }^{22}$. A logical next step would be to delete other murine cytochrome clusters in this model, but considering that there are 72 functional genes and probably a similar number of pseudogenes in the murine genome, this would be a major challenge.

Despite the magnitude of the cytochrome P450 family, all cytochromes have only one electron donor, the NADPH-P450 oxidoreductase (Por). Deletion of the Por gene is embryoniclethal (E10.5) because the enzyme is involved in a variety of essential metabolic pathways ${ }^{24}$. Nevertheless, liver-restricted deletion of Por leads to healthy mice with some metabolic deficiencies in cytochrome, cholesterol, and heme metabolism ${ }^{25-27}$.

To develop a better animal model for human-specific drug metabolism, we generated a conditional Por mouse and deleted the I 2 rg, Rag2 and Fah genes in the homozygous zygotes. We show that this PIRF strain is readily repopulated with human hepatocytes and reflects human xenobiotic metabolism upon deletion of the murine Por gene. Thus, these PIRF mice could be used to predict human drug metabolism and should improve preclinical drug development.

\section{Results}

Generation of novel mouse model for hepatocyte repopulation. In order to functionally block murine cytochrome metabolism, we generated a conditional (floxed exon 3 and 4) knock-out of the NADPH-P450 oxidoreductase (Por) gene by targeting mouse embryonic stem cells (ESCs) ${ }^{28}$ (Supplementary Fig. 1). Injected blastocysts with properly targeted ESCs produced chimeras with germline transmission of the Por "knock-out first" allele ${ }^{29}$. We confirmed expression from the targeted Por locus using the lac $Z$ expression cassette in the embryo and adult liver (Supplementary Fig. 1c). We next bred the mice with a flippase-expressing strain ${ }^{30}$ to generate a CRE recombinase conditional Por knock-out strain $\left(\right.$ Por $\left.^{c / c}\right)$. Homozygous zygotes from this strain were injected with the bacterial type II clustered regularly interspaced short palindromic repeats/Cas9 (CRISPR-Cas9) system ${ }^{31-33}$ targeting simultaneous deletion of critical exons of the Il2rg, Rag2, and Fah genes (Supplementary Figs 2 and 3) to generate the PIRF strain (Fig. 1a). Homozygous PIRF mice are thus immune-deficient, lacking T, B, and NK cells, but are healthy and fertile. Since adenoviral gene therapy vectors efficiently transduce hepatocytes in vivo, we deleted the Por gene using an adenovirus coding CRE recombinase (Adeno-CRE). We injected increasing doses $(2.2 \times$ $10^{8-10}$ per mouse) of the virus intravenously into PIRF mice. Quantitative RT-PCR of the Por mRNA in liver revealed efficient deletion only at high doses of adenovirus (Fig. 1b). Immunostaining for Por (Fig. 1c) confirmed these findings, although a minimal residual signal could be detected by western blotting even at the highest dose used (Fig. 1d). Por-deleted PIRF mouse livers accumulated lipids starting about 2 weeks after adenoviral transduction (Fig. 2a), but in contrast to the immune competent $\mathrm{Alb}-\mathrm{Cre} / \mathrm{Por}^{\mathrm{c} / \mathrm{c}}$ strain $^{25}, 27$, without infiltration and lacking necrosis (Fig. 2b). Nevertheless, residual Por expressing hepatocytes had a growth advantage over the lipid-rich Por-deleted hepatocytes, and clonal expansion of a few Por expressing cells could be detected 4 weeks after adenoviral transduction by immunostaining (Fig. 2c).

Characterization of humanized PIRF mice. We next generated human liver chimeric mice using the PIRF strain ${ }^{5}, 20,34$. To ensure cytochrome P450 metabolism would be human-specific, we injected Adeno-Cre $\left(2.2 \times 10^{10}\right.$ pfu per mouse $)$ before human hepatocyte transplantation and an additional dose of Adeno-Cre in some highly humanized PIRF (Hu-PIRF) mice. Immunostaining revealed that an almost complete deletion of the Por gene could be achieved only in double-injected humanized PIRF (Hu-PIRF 2x) mice (Fig. 3a). Quantitative PCR and western blotting corroborated the massive reduction of murine Por upon adenoviral delivery of CRE (Supplementary Fig. 4). We next performed gene expression profiling to compare PIRF mice repopulated with human hepatocytes (Hu-PIRF) that were injected with either Adeno-CRE (Hu-PIRF 2x) or Adeno-GFP (Fig. 3b). Both groups were repopulated with human hepatocytes from the same hepatocyte donors (Supplementary Table 1) to avoid inter-individual variations.

Expression of the murine P450 cytochromes was clearly altered for 27 out of 38 genes analyzed after Por deletion (Fig. 3c): 24 cytochromes were significantly upregulated (1.5-12.5-fold) and 3 cytochromes significantly downregulated (0.5-0.3-fold). The expression profiles of these murine cytochromes were by in large comparable to those from previous work in non-humanized, Pordeficient mice (Supplementary Table 2$)^{35}$. In the human part of the same chimeric liver, human P450 cytochromes were less altered upon deletion of murine Por (Fig. 3d). Half of the human cytochromes were only slightly altered (0.5-1.5-fold change), while the other half were moderately upregulated (1.5-2.4-fold).

Not all human cytochromes serve an important role in xenobiotic metabolism. From the 200 most-prescribed drugs in the United States, about three-quarter are metabolized through P450 cytochromes, of which CYP3A4/5, 2C9, 2C19, 2D6, and $1 \mathrm{~A} 2$ contribute to $\sim 95 \%{ }^{36}$. We compared these human cytochrome clusters from chimeric livers (Hu-PIRF $2 x$ ) with the originating, isogenic primary hepatocytes. For this comparison, we again used two donor hepatocytes (Supplementary Table 1) and the corresponding human (isogenic) liver chimeric 
mice $(N=6)$. Expression levels were similar for most clusters, and these important cytochromes were all robustly expressed in chimeric livers (Fig. 3e). Interestingly, some human clusters (CYP1A2, CYP2B6, CYP2C19, and CYP3A4) were expressed at even higher levels in the chimeric liver than in primary human hepatocytes.

Xenobiotic metabolism of humanized PIRF mice. To validate $\mathrm{Hu}$-PIRF mice for human drug metabolism, we studied xenobiotic metabolism of gefitinib ${ }^{37}$, an inhibitor of epidermal growth factor receptor used against lung cancer and a variety of other neopla$\mathrm{sia}^{38}$. Gefitinib is metabolized primarily by the $\mathrm{P} 450$ cytochrome system, including CYP3A4 and 2D6. We recently identified new gefitinib metabolites and demonstrated considerable differences between human and mouse liver microsomes ${ }^{39}$, but regardless of dose, route, or species, gefitinib is excreted primarily in the feces $(<7 \% \text { in the urine })^{40,41}$. We therefore analyzed the feces of nonhumanized PIRF mice for gefitinib metabolites during the first 16 $h$ after intravenous injection of gefitinib.

Mass spectrometry revealed a reduction of several gefitinib metabolites upon deletion of the Por gene, implying a

a
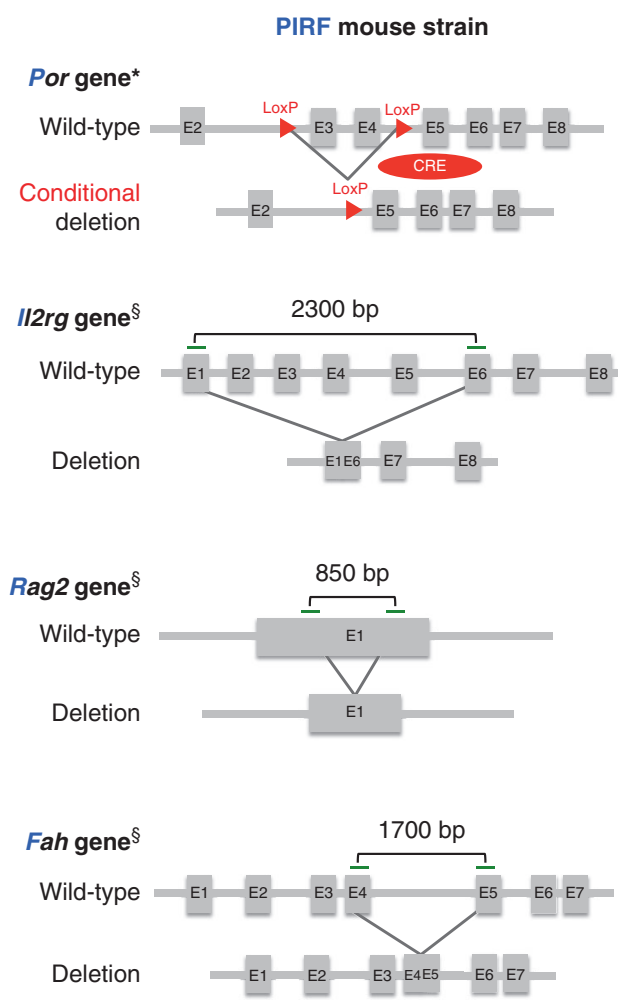

*Embryonic stem cell targeting (Supplementary Fig. 1)

§zygote injection with Cas9 and gRNA

(Supplementary Fig. 2\&3)
Por-dependent P450 cytochrome deficiency for these metabolites (Fig. 4a and Supplementary Fig. 5). Since some metabolites were not significantly altered, we tested the possibility that residual Por activity was responsible for persistent murine P450 cytochrome metabolism in our system. We crossed the $\mathrm{Por}^{\mathrm{c} / \mathrm{c}}$ strain with a transgenic mouse that expresses CRE under the albumin promoter. Por protein in the liver of $A l b-C R E / \mathrm{Por}^{\mathrm{c} / c}$ animals was efficiently deleted (Supplementary Fig. 6); nevertheless, the metabolite profile formed after gefitinib injection was comparable to that of PIRF mice with adenoviral deletion of Por (Supplementary Fig. 5). This result similarity indicates that gefitinib has both P450-dependent and -independent drug metabolism.

The biggest and most relevant reduction was observed for O-desmethyl gefitinib (M4, M523595), which is by far the most abundant metabolite in human feces. Rodents produce many different metabolites in addition to $4^{40,41}$ (Fig. 4b), so we analyzed the M4 metabolite in murine Por-deleted and Por-expressing humanized and non-humanized control mice (Fig. 4c). The highest levels of M4 were detected in murine Pordeficient Hu-PIRF mice, where human hepatocytes preferentially

b
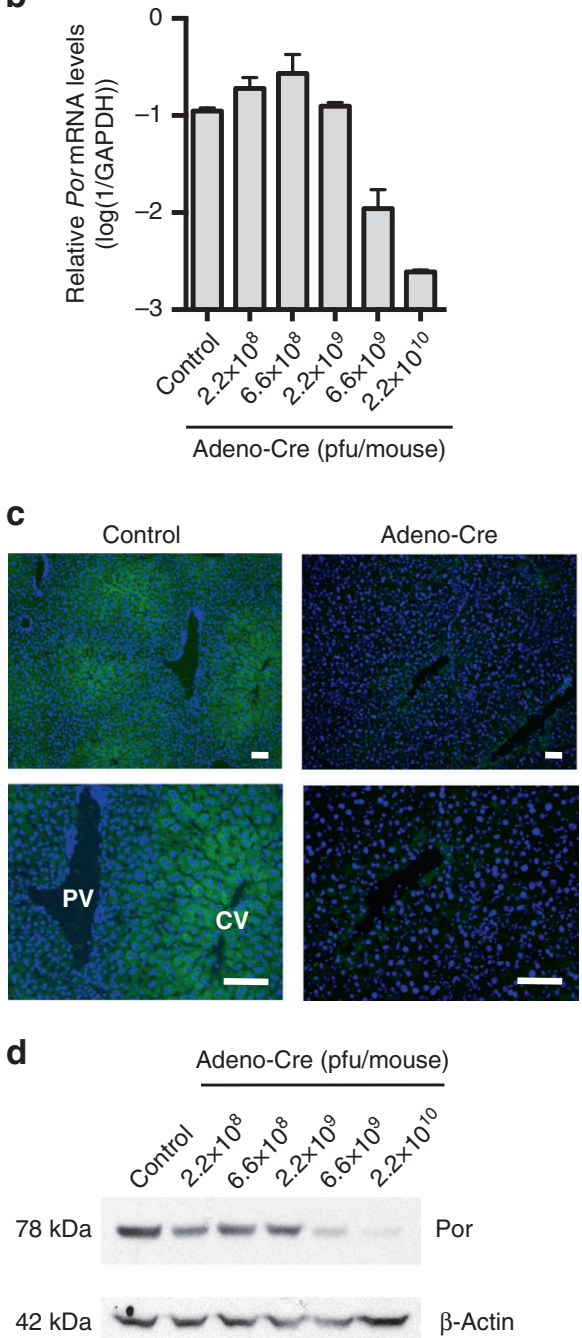

Fig. 1 Generation of the PIRF strain and deletion of the murine P450 (Por) oxidoreductase. a Schematic representation of deleted and transgenic loci in the PIRF strain. $\mathbf{b}$ qPCR of Por mRNA 7 days after intravenous injection of adenovirus expressing the CRE recombinase (Adeno-Cre). $\mathbf{c}$ Immunostaining for Por protein demonstrating a gradient across the hepatic acinus with higher pericentral (cv) and lower periportal (pv) expression. Seven days after injection with Adeno-Cre, Por is barely detectable. $\mathbf{d}$ Western blotting confirms almost complete disappearance of Por protein 7 days after injection. Results are expressed in mean values \pm s.e.m. of triplicates $(\mathrm{N}=3)$. Scale bar $50 \mu \mathrm{m}$. PIRF, Porc/c $/ 112 \mathrm{rg}^{-/-} / \mathrm{Rag}^{-/-} / \mathrm{Fah}^{-/-}$ 
a
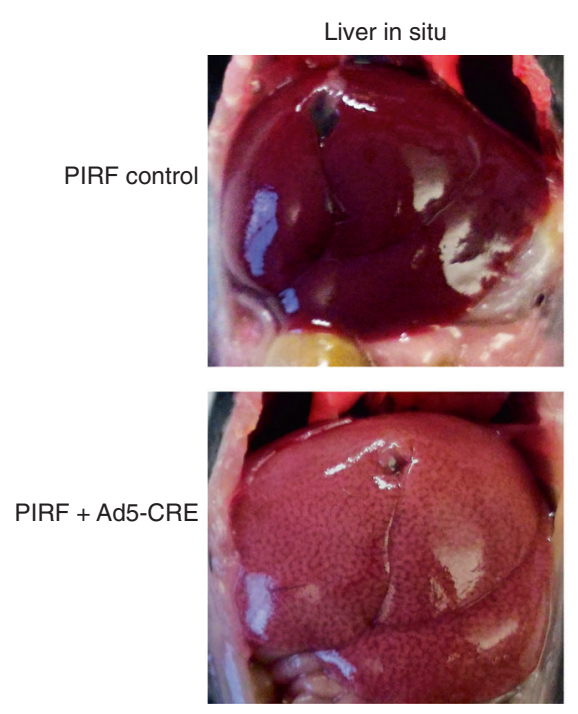

b

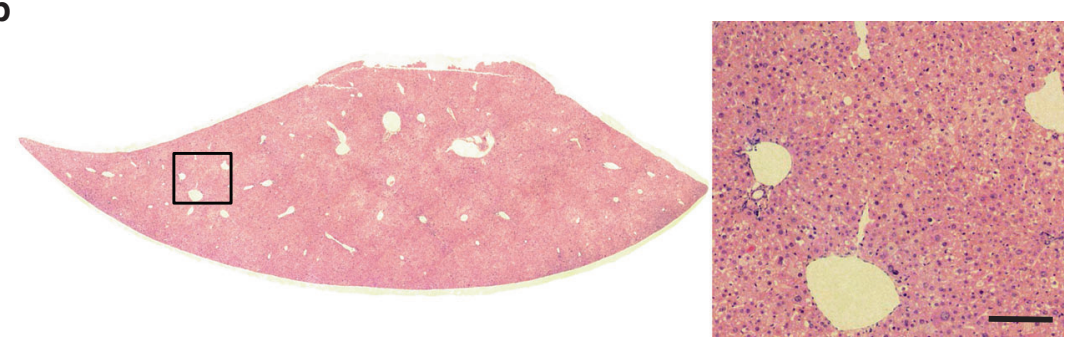

C
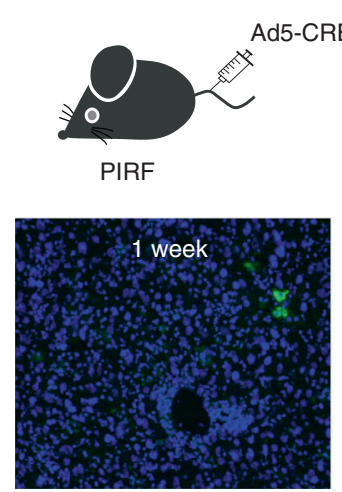

Oil-Red-O staining
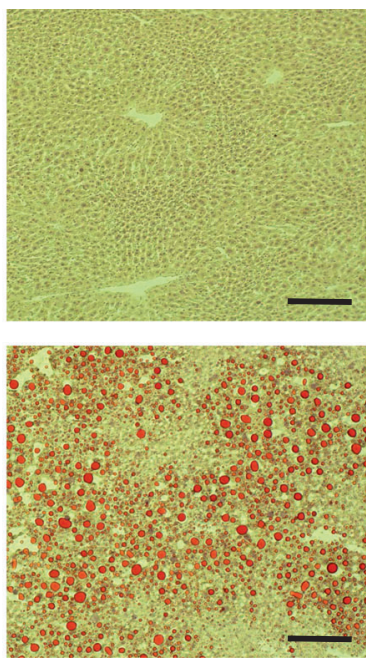
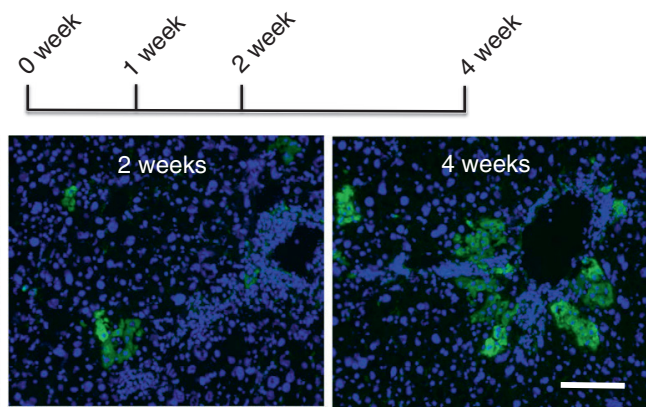

Fig. 2 Deletion of Por with an adenoviral vector expressing CRE recombinase in PIRF mice. a 2 weeks after injection of the adenovirus $\left(2.2 \times 10^{10}\right.$ pfu per mouse) hepatocytes start to accumulate lipids. $\mathbf{b}$ H\&E stain of liver lobe 4 weeks after transduction with adenovirus showing macro and microvesicular steatosis. Right picture is a higher magnification of boxed area on left. c Clonal expansion of Por expressing hepatocytes in PIRF mice. Mice were injected

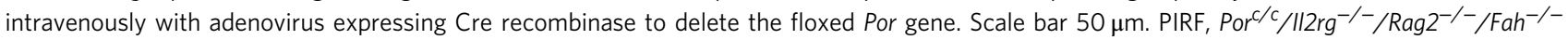

metabolize gefitinib to M4 and the remaining murine hepatocytes are inhibited in their drug metabolism (Fig. 4d). We next sought to measure other human-specific metabolites. The most abundant human metabolite was M28, which could not be detected at all in our non-humanized control mice. Mass spectrometry again showed the highest level of this human-specific metabolite in murine Por-deficient Hu-PIRF mice (Fig. 4e, Supplementary Fig. 7), confirming that these mice showed liver metabolism more similar to humans.

The Por-deficient Hu-PIRF mouse is a novel model system for drug metabolism studies, and we therefore analyzed different body compartments, e.g. the serum ( $1 \mathrm{~h}$ after injection) and the urine for these key gefitinib metabolites. M4 could not be detected in the urine and was massively reduced (23-fold in Hu-PIRF mice) in the serum, while M28 was detectable at lower concentrations in both the urine and the serum of Hu-PIRF mice (Supplementary Fig. 8a, b). Although present at lower levels in both compartments, M28 mirrored relative abundance observed in feces (Fig. 4d). These findings confirm that gefitinib metabolites are primarily excreted trough the feces ${ }^{40,41}$.

We next sought to confirm human xenobiotic metabolism using liver homogenates of PIRF mice. This time we tested atazanavir, an antiretroviral drug (protease inhibitor) for treatment of human immunodeficiency virus. Our previous studies in human and mouse microsomes demonstrated that atazanavir metabolite M15 is a predominant human metabolite ${ }^{42}$. To determine levels of M15 in humanized PIRF mice, we intravenously injected them with atazanavir and harvested their livers 30 min after injection. M15 levels in Por-deleted humanized PIRF mice were 5.4 times greater than those observed in nondeleted mice (Fig. 4f), again indicating that these mice metabolize drugs as humans do. 
a

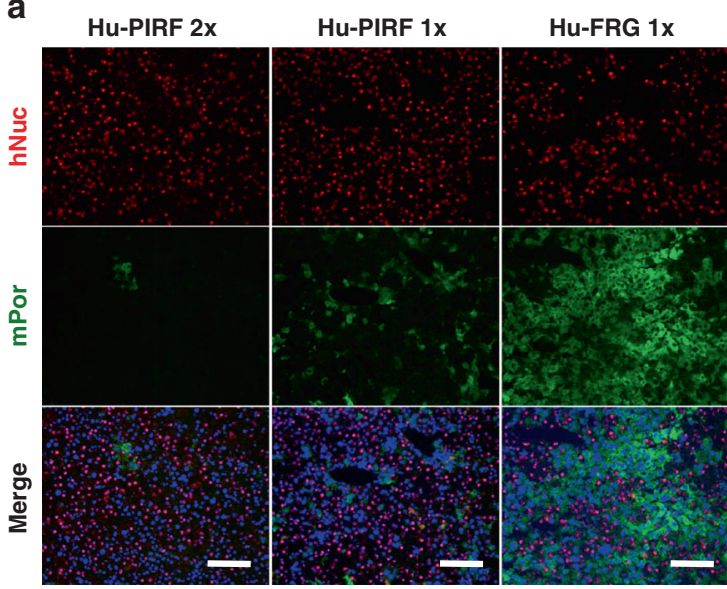

d

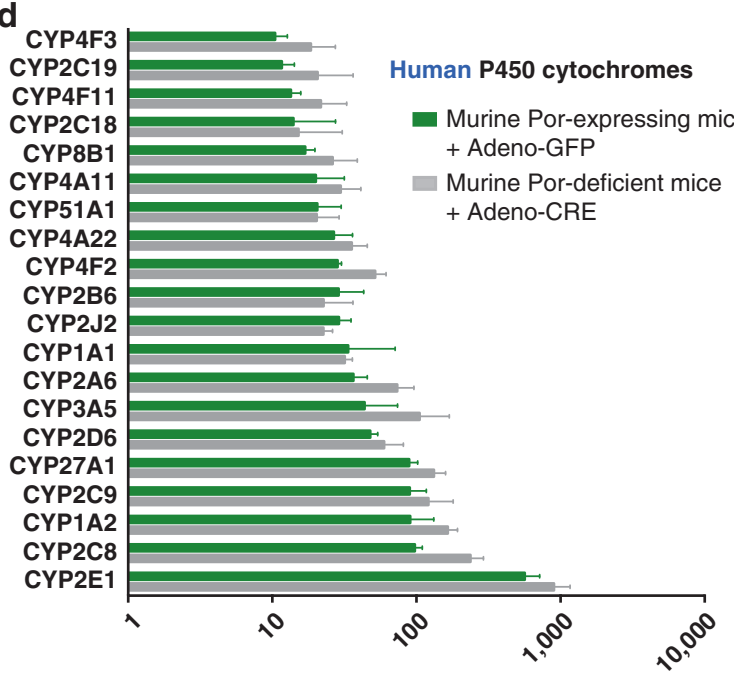

Relative mRNA expression (normalized to four housekeeping genes)

e

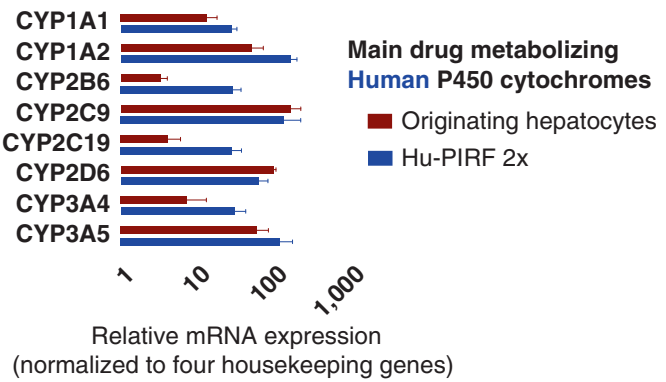

b

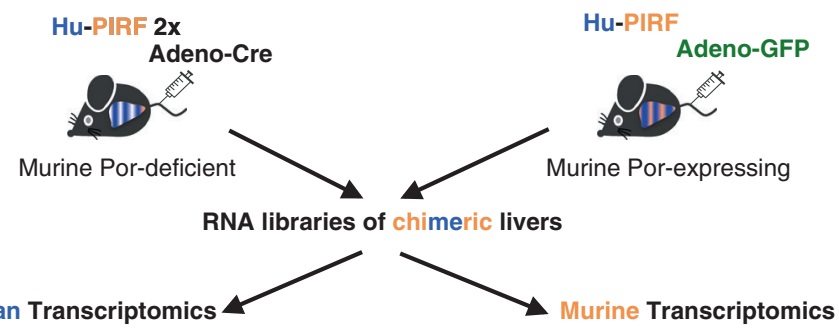

c

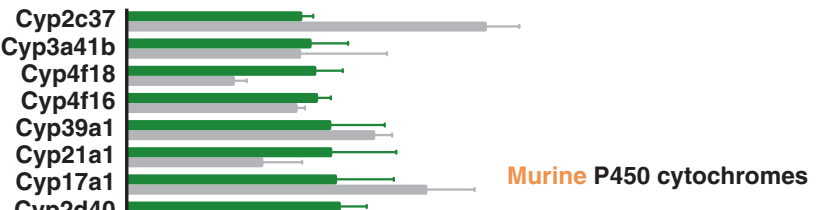

Murine Por-expressing mice + Adeno-GFP

Cyp4f14

Cyp4f13

Cур2c67

Cyp4a10

Cyp4f15

Cyp2c38
Cyp2j6

Cyp2j6
Cyp2d22

Cyp2c68

Cyp4a14

Cyp3a25

Cyp3a13

Cyp4v3

Cyp3a41a

Cyp2j5

Cyp27a1

Cyp2c39

Cyp2c29

Cyp1a2

Cyp2a12

Cyp2d9

Cyp2d10

Cyp2e1

Cyp2c70

Cyp2d26

Cyp3a11

Cyp2a4

Cyp2a5

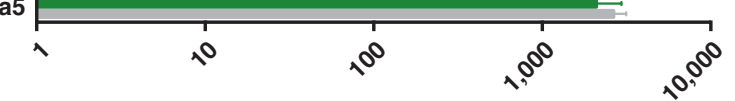

Relative mRNA expression (normalized to four housekeeping genes)

Fig. 3 Gene expression profiling of humanized PIRF mice following deletion of Por. a Immunostaining of humanized PIRF and FRG mice for murine Por (mPor) and human nuclei (hNuc) after injection of Adeno-Cre $\left(2.2 \times 10^{10} \mathrm{pfu} /\right.$ mouse) once $(1 \mathrm{x})$ or twice (2x). Counterstaining in merged picture using DAPI. b Experimental outline for murine and human transcriptomics from chimeric livers with or without Por deletion. Chimeric mice $(N=7)$ injected with either Adeno-Cre or Adeno-GFP (2.2 $\times 10^{10} \mathrm{pfu}$ /mouse) were euthanized after 1 week and liver tissue from all seven lobes was analyzed. Comparison of murine $\mathbf{c}$ and human $\mathbf{d}$ cytochromes originating from the same chimeric livers. $\mathbf{e}$ Gene expression of the main drug metabolizing human cytochromes in humanized, Por-deleted PIRF (Hu-PIRF 2x) mice and originating isogenic human hepatocytes ( $N=2$ donor hepatocytes and 4 isogenic humanized mice). Gene expression has been normalized to four human housekeeping genes and their murine counterparts (PSMB2, PSMB4, RAB7A, and VPS29; Psmb2,

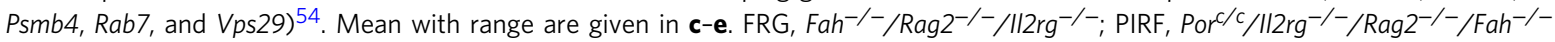

\section{Discussion}

Identification of (reactive) metabolites is crucial for establishing the safety of new drugs, since metabolites drive human drug toxicity ${ }^{43}, 44$. Early and accurate detection of human metabolites is important to mitigate risk and optimize the development of a chemical compound into a drug. Primary assessment of human drug metabolites is usually done using three in vitro systems derived from human liver: liver microsomes, S-9-fractions, or plated human hepatocytes. Each system can predict primary metabolites fairly well, but is limited in predicting more complex multi-step biotransformations ${ }^{45}$. In addition to this major limitation, there is a need for rigorous standardization, since many 
experimental parameters such as the incubation time of the drug or protein concentration can bias the analysis ${ }^{46}$. Human hepatocyte-derived in vitro systems are a good first step but cannot replace ADME studies. From an ethical perspective, animal studies are the next step, before moving into clinical trials. The drawback is that experimental animal models have a divergent, usually expanded and poorly characterized set of drug metabolizing enzymes. With the advent of human liver chimeric mice, a new and attractive option was available to study human drug metabolism in vivo without endangering any humans.

Here we introduce a next generation of humanized mouse model amenable to human drug metabolism with minimal interference from the murine P450 cytochromes. We compared the production of human metabolites for two different drugs a

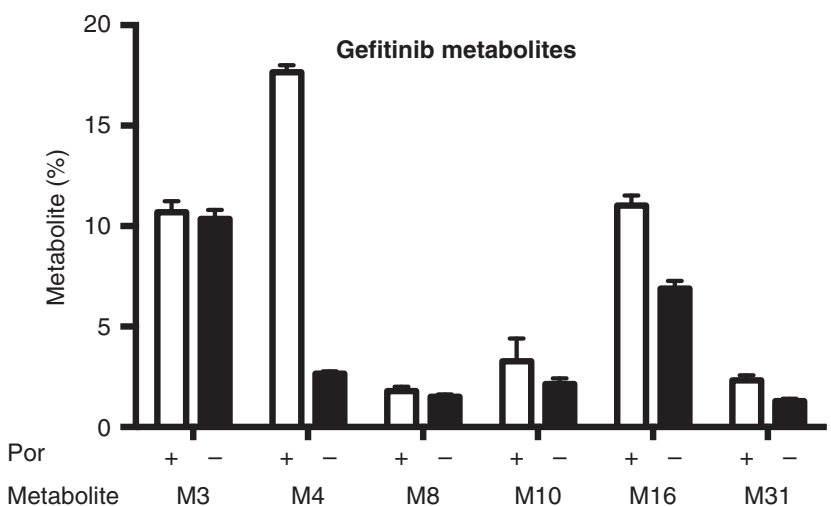

C

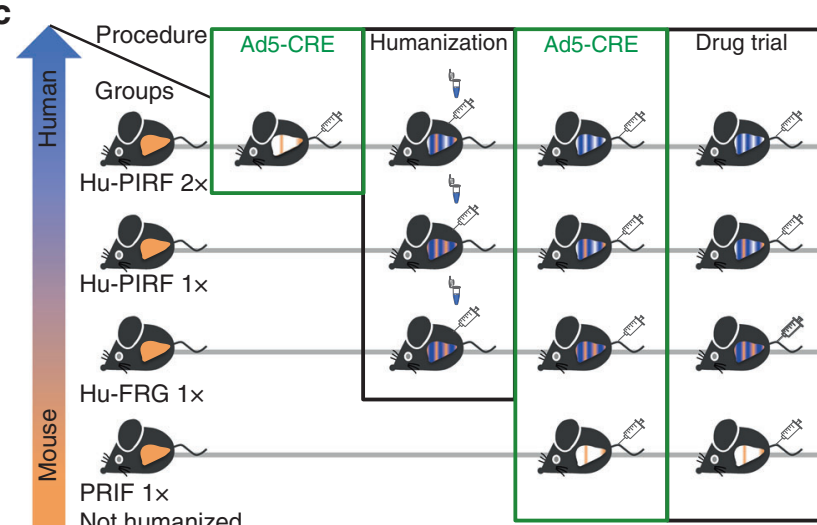

Human specific metabolite - M28

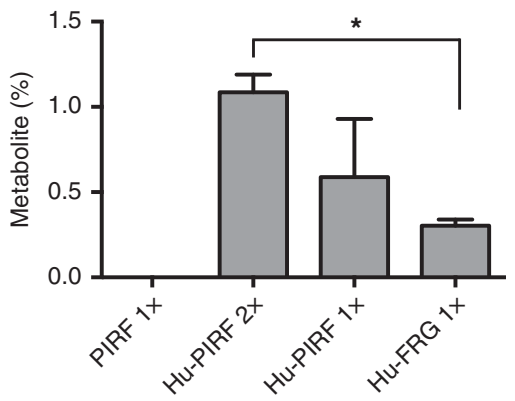

b

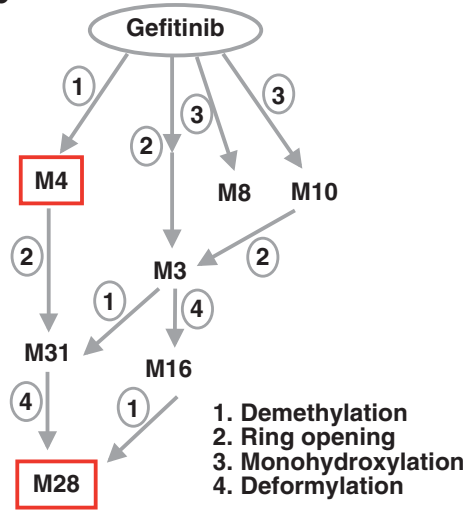

d

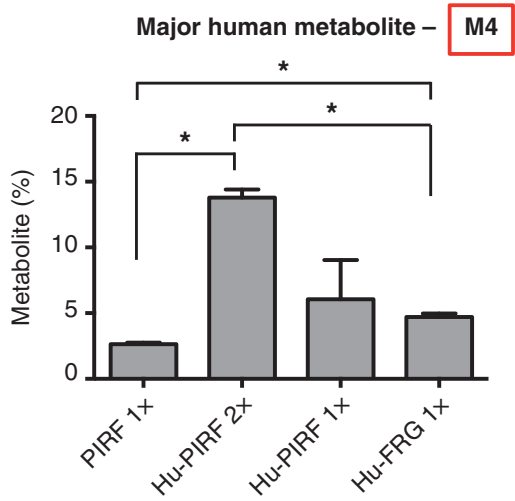

f

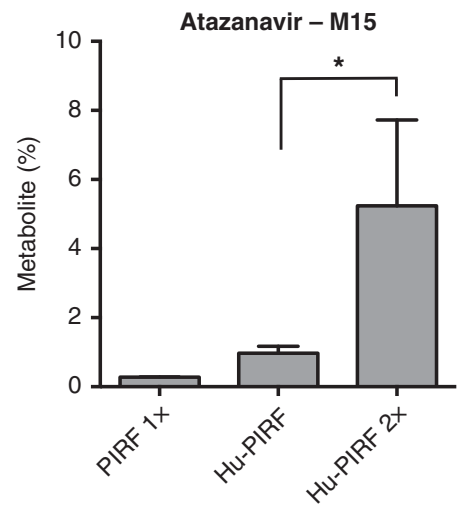

Fig. 4 Xenobiotic metabolism in humanized PIRF mice. Mass spectrometry analysis of murine feces within $16 \mathrm{~h}$ after intravenous injection of gefitinib a cancer therapeutic. a Selection of abundant and decreased gefitinib metabolites upon murine P450 oxidoreductase (Por) deletion in non-humanized PIRF mice $(N=3)$. b Gefitinib metabolites and known biotransformation. c Experimental setup: not-humanized PIRF mice and Humanized (Hu) FIRF and FRG mice were injected once ( $1 \mathrm{x}$, after transplantation of human hepatocytes) or twice (2x, before transplantation and after reaching high human chimerism) before doing drug studies. All experimental groups contain three animals, if humanized, with high (>70\%) and similar human chimerism (see Methods). Murine Por-deleted, human liver chimeric PIRF (Hu-PIRF 2x) mice show the most abundant human metabolite, M4 d, and the human-specific metabolite M28 e of gefitinib $(\mathrm{N}=3)$. f Mass spectrometry analysis of PIRF liver homogenates 30 min after injection of atazanavir, a retroviral therapeutic. A major human metabolite (M15) is shown. The overall abundance of metabolites from atazanavir or gefitinib was set as $100 \%$ in each sample. The data are

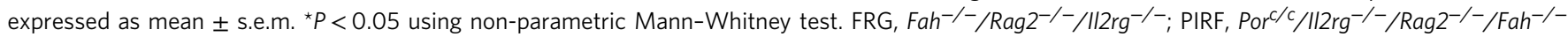


between humanized PIRF mice and "normal" humanized FRG mice. Analyses revealed higher concentrations of human metabolites in murine feces and liver homogenate in humanized PIRF mice than in FRG mice and demonstrate that these mice have humanized drug metabolism. The PIRF and FRG strains used in this study are in a mixed (C57B and 129 S) genetic background. Aside from potential differences in the background to the two previously published FRG mouse strains 5,7 , our CRISPR/Cas9 generated knock-out strains do not express any transgenes, e.g. the neomycin phosphotransferase, that inactivates a wide range of aminoglycoside antibiotics. This model system should be useful for early detection of reactive metabolites and shape early drug development approaches. Our results are encouraging, and while this is an elegant way to block a large and confounding cluster of drug metabolizing murine enzymes, there is room for improvement. First, adenovirus mediated Por deletion occurs primarily in the liver, which is the major but not the only site of cytochrome metabolism. Although a complete Por knock-out is embryonic-lethal and might not be compatible with life even after development, knocking out Por in a combination of multiple organs like the gut and the liver or the lung and the liver would be desirable. Another consideration is that drug metabolism is not limited to cytochrome metabolism, and additional deletions in other drug-metabolizing enzymes could improve this system. Lastly, it is conceivable that Por deletion could be achieved more efficiently. Using transgenic mice expressing Cre recombinase would require yet another crossing step into a quadruple transgenic (PIRF) mouse, however, and an early organ-specific deletion might not generate a robust strain amenable to xenotransplantation.

In summary, we have generated a novel mouse model combining human chimerism with functional deletion of all murine cytochromes by Por deletion. Such a murine Por-deficient humanization could also be used in combination with other repopulation models such as the transgenic uPA mouse ${ }^{11,21}$. Our data with two different drugs in two different body compartments demonstrate that studies in humanized PIRF mice efficiently identify human metabolites. Future work will determine how robust this novel system is and demonstrate its limitations. These next-generation human liver chimeric mouse models should promote the development of safer and more efficacious drug therapies.

\section{Methods}

Generation of the Por-floxed mouse strain. Por knock-out first targeting vector was purchased from the National Institutes of Health Knock-Out Mouse Program (Supplementary Fig. 1). The vector was linearized with the AsisI restriction enzyme, and DNA was electroporated into Jm8A3 mouse $\mathrm{ESCs}^{47}$ by the Mouse Embryonic Stem Cell Core at Baylor College of Medicine. Integrated clones were selected using neomycin resistance. DNA of ESC clones was digested with NSiI restriction enzyme and screened for site-specific integration by Southern blotting using DIG nonisotopic detection system (Roche Applied Biosciences) following the manufacturer's instructions (full blots in Supplementary Fig. 9). The $500 \mathrm{bp}$-size $5^{\prime}$ and $3^{\prime}$ probes that bind outside the vector's homology arms were synthesized using the following set of primers:

\section{5'-Por Fw2 GGCCTCAGAGAGGACATAGTGCCC-3' \\ 5'-Por Rev2 GCCCTCTGGTGTCAGGTCCC-3' \\ 3'-Por Fw2 CCTCACGCAGCTTAATGTGGCC-5' \\ 3'-Por Rev2 GGAAGTTAAGGACGTGATTACAGGGAGC-5'}

Correctly targeted ESCs cells were injected into C57/BL blastocysts by the Genetically Engineered Mouse Core at Baylor College of Medicine. The male chimeras were bred with C57/BL albino females (Taconic) to visualize germline transmission of targeted ESC. To remove the FRT-flanked LacZ and the neomycin cassette and generate a conditional Por knock-out strain $\left(\mathrm{Por}^{\mathrm{c} / \mathrm{c}}\right)$, we crossed the mice with a Rosa26-FLPe strain ${ }^{30}$. Genotyping was performed by Transnetyx (Cordova, TN).

X-Gal staining. Embryos and fresh liver sections were fixed in $4 \%$ PFA for $1 \mathrm{~h}$ at at $4^{\circ} \mathrm{C}$ and washed $2 \times 30 \mathrm{~min}$ in X-Gal rinse buffer (PBS $1 \times$ with $0.02 \%$ Igepal and
$0.01 \%$ deoxycholate) followed by overnight incubation with X-Gal staining solution (PBS $1 \times$ with $5 \mathrm{mM} \mathrm{K}_{3} \mathrm{Fe}(\mathrm{CN})_{6}, 5 \mathrm{mM} \mathrm{K}_{4} \mathrm{Fe}(\mathrm{CN})_{6}, 0.02 \%$ Igepal, $0.01 \%$ deoxycholate, $2 \mathrm{mM} \mathrm{MgCl}$, $5 \mathrm{mM}$ EGTA, and $1 \mathrm{mg}$ per $\mathrm{ml}$ of fresh X-Gal). Samples were post-fixed overnight in $4 \%$ PFA at $4^{\circ} \mathrm{C}$

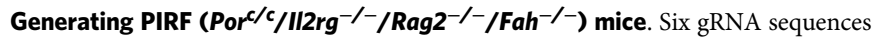
targeting critical exons of the Rag2, Il2rg, or Fah gene were selected (Fig. la, Supplementary Figs 2 and 3) using two different online tools (crispr.mit.edu and $\operatorname{COSMID}^{48}$ ). Complementary oligonucleotides were annealed and ligated into the DR274 vector ${ }^{49}$ (Addgene plasmid \# 42250) using standard molecular cloning techniques with the restriction enzyme BsaI (NEB) and T4 DNA Ligase (NEB). A T7 bacterial promoter sequence was inserted into the pX330-U6-Chimeric_BBCBh-hSpCas9 vector ${ }^{50}$ (Addgene plasmid \# 42230) upstream of the Cas9 transcription start site using standard molecular cloning techniques. DR274 vectors were cut using DraI (NEB) and gelpurified using the Zymoclean Gel DNA Recovery Kit (Zymo, Cat\#11-301). In vitro transcription of sgRNA was performed using the MEGAshortscript T7 Transcription Kit (Life Technologies, AM1354) according to manufacturer's instructions. The resulting RNA was purified using the RNA Clean \& Concentrator-5 (Zymo, R1015) and eluted in RNAse-free water Synthesis was verified by polyacrylamide gel electrophoresis. pX330 (with T7 promoter) was digested with NcoI and NotI, and gel purified. Cas9 mRNA was synthesized from the digested $\mathrm{pX} 330-\mathrm{T} 7$ vector using the mMessage mMachine T7 ULTRA Kit (life tech AM1345), according to the manufacturers protocol. Polyadenylation was verified by denaturing agarose gel electrophoresis ( $1 \%$ agarose and $6.6 \%$ formaldehyde in MOPS buffer).

Zygotes from Por ${ }^{c / c}$ mice were injected with $S$. pyogenes Cas 9 mRNA $(60 \mathrm{ng} / \mathrm{ul})$ and the six gRNA ( $15 \mathrm{ng} / \mathrm{ul}$ each). All viable zygotes were implanted into three pseudopregnant females. To detect the deleted regions, all 23 pups were genotyped after weaning using the following primers:

\section{Fah Fw CTGGGTTGCATACTGGTGGG \\ Fah Rev AAACAGGGTCTTTGCTGCTG \\ Fah Int Fw ACAAAGGTGTGGCAAGGGTT \\ Il2 Fw CCACCGGAAGCTACGACAAA \\ Il2 Rev GGGGGAATTGGAGGCATTCT \\ Il2 Int Rev CTTCTTCCCGTGCTACCCTC \\ Rag2 Fw CCTCCCACCTCTTCGTTATCC \\ Rag2 Rev AGTCTGAGGGGCTTTTGCTA \\ Rag2 Int Fw AGTCTGAGGGGCTTTTGCTA}

Further offspring genotyping was performed by Transnetyx (Cordova, TN).

Humanization of PIRF mice. Hepatocytes $\left(3 \times 10^{6}\right.$ per mouse) purchased from Triangle Research Labs/Lonza were transplanted into the murine liver of female PIRF mice by splenic injection, as originally described for mouse hepatocytes ${ }^{51}$. In brief, the abdominal cavity was opened by a midabdominal incision, and $3 \times 10^{6}$ human hepatocytes in a volume of $100 \mu \mathrm{l} \mathrm{HCM}$ were injected into the spleen. Immediately after transplantation, selection pressure toward transplanted human hepatocytes was applied by withdrawing the drug nitisinone (NTBC) from the drinking water in the following steps: 2 days at $25 \%$, then 2 days at $12 \%$, and eventually 2 days at $6 \%$ of the colony maintenance dose $(100 \%=7.5 \mathrm{mg} / \mathrm{l})$ prior to discontinuing the drug completely ${ }^{20}$. Mice with clinical symptoms (hunched posture, lethargy, weight loss, etc) were put back on $100 \%$ nitisinone for a few days before once again being weaned off the drug as described above. In order to determine the extent of human chimerism, we measured human albumin (ELISA, Bethyl laboratories) in the murine blood, having previously shown that human albumin levels correlate with the level of human chimerism assessed by immunostaining of human hepatocytes ${ }^{20}$. Only mice with a human chimerism $>70 \%$ were used for this study. Where indicated, some PIRF mice were injected intravenously with $100 \mu \mathrm{l}$ Adenovirus coding CRE recombinase under the CMV promoter (Ad5 CMV-Cre, $2.2 \times 10^{10} \mathrm{pfu} / \mathrm{ml}$, provided by the Vector Development Laboratory at Baylor College of Medicine) either $24 \mathrm{~h}$ before hepatocyte transplantation and/or when reaching high human chimerism (>70\%). Available hepatocyte donor information is given in Supplementary Table 1. All animal experiments were approved by the Baylor College of Medicine Institutional Animal Care and Use Committee. All animals used for humanization (including controls) were female, due to fewer postsurgical complications.

qPCR. Total mRNA was isolated from fresh frozen tissue samples using Purelink RNA mini kit (Invitrogen). Two micrograms of total mRNA was reversetranscribed using the qScript cDNA supermix (Quanta Biosciences) and $20 \mathrm{ng}$ of cDNA was used for the qPCR reactions, performed with Perfecta SYBR Green Fast Mix (Quanta Biosciences) and analyzed on ABI Prism 7900HT Sequence Detection System (Applied Biosciences). The following primers were used for Por mRNA amplification of PIRF mouse samples:

\section{$m$ Por Fw2 GGCCCCACCTGTCAAAGAGAGCAGC}

mPor Rev1: CAAACTTGACACCCGTGAGGTCC

For humanized PIRF mouse liver samples, mouse Por and human POR were amplified using the following set of primers: 
mPor Fw1: TCTATGGCTCCCAGACGGGAACC $m$ Por Rev2: CCAATCATAGAAGTCCTGCGCG hPOR Fw1: CCAATCATAGAAGTCCTGCGCG hPOR Rev5: ACCTTGGCCGCATCTATGTCGG

Each sample was normalized to Gapdh/GADPH as an internal control gene using the following primers:

$m$ Gapdh Fw AGAACATCATCCCTGCATCCA

$m G a p d h$ Rev CAGATCCACGACGGACACATT

$h G A P D H$ Fw: CAGAACATCATCCCTGCCTCTAC

$h G A P D H$ Rev: TTGAAGTCAGAGGAGACCACCTG

RNA-Seq libraries. Whole-transcriptome RNA sequencing (RNA-Seq) was performed using total RNA extracted from fresh-frozen liver tissue sampled from all seven liver lobes. Total RNA was isolated using the Purelink RNA mini kit (Invitrogen). Libraries were generated from total RNA according to the manufacturer's recommendation using the TrueSeq Stranded mRNA LT kit (Illumina). The libraries were sequenced on a NextSeq 500 sequencer. The average read count per sample was 32 million. RNA-Seq TPM expression values were calculated with RSEM $^{52}$ (version 1.2.17) using the read aligner Bowtie $2^{53}$ applied to the combined human and mouse NCBI Refseq (3/21/16) transcriptomes. Low-abundance cytochromes (human $<20$ TPM and mouse $<20$ TPM) were only compared if one of the experimental groups reached $>20 \mathrm{TPM}$. Gene expression has been normalized to four human housekeeping genes and their murine counterparts (PSMB2, PSMB4, RAB7A, and VPS29; Psmb2, Psmb4, Rab7, and Vps29) ${ }^{54}$. RNA-Seq data is available from European Nucleotide Archive, ENA accession code PRJEB14714.

Western blot. Tissue from snap-frozen liver was homogenized in RIPA buffer (Sigma, cat\# R0278-50 ml) containing protease inhibitors (Roche, cat\# 04693159001). Thirty micrograms of total protein was electrophoresed in a NuPAGE 4-12\% Bis Tris Gel (Invitrogen, cat\# NP0336BOX) and transferred to a PVDF membrane (Millipore, cat\# IPVH00010). The blot was then blocked in $5 \%$ milk, followed by primary antibody incubation. Rabbit anti-Por (Abcam cat\# ab13513) or mouse anti- $\beta$-actin (Sigma cat\# A1978) were diluted 1:1,000 and $1: 3,000$, respectively (full blots in Supplementary Figs 10 and 11). Secondary antibodies were donkey anti-rabbit IgG/HRP and donkey anti-mouse IgG/HRP (Jackson Immunoresearch Labs, cat\# 711-035-152 and 711-035-150) used at $1: 10,000$ and 1:50,000, respectively. The membrane was imaged using Amersham ECL Western Blotting Detection Reagent (General Electric Healthcare Life Sciences, cat\# RPN2106).

Immunohistochemistry. Ten-micrometer sections from cryopreserved tissue blocks were fixed with $3 \%$ PFA for $15 \mathrm{~min}$ and incubated overnight at $4{ }^{\circ} \mathrm{C}$ with the following primary antibodies: anti-Por (Abcam, cat\# ab13513) diluted 1:500, antihuman Nuclei (EMD Millipore, cat\# MAB1281) diluted 1:250 in PBS containing $0.2 \%$ Triton X-100, and $0.5 \%$ BSA. Secondary antibodies (1:1,000 Alexa-fluor conjugated, Molecular Probes) were incubated for $60 \mathrm{~min}$ at room temperature in the same buffer. Sections were mounted with Vectashield plus DAPI (Vector Labs).

Sample preparation for mass spectrometry. Female mice (6- to 10 -month old, humanized or non-humanized) were maintained under a standard 12-h dark/light cycle with water and chow provided ad libitum. The mice were treated (i.v.) with gefitinib $(10 \mathrm{mg} / \mathrm{kg})$ and housed separately in metabolic cages for $16 \mathrm{~h}$ feces collection. Feces samples were weighed and homogenized in water $(100 \mathrm{mg}$ feces in $1,000 \mu \mathrm{l}$ of $\mathrm{H}_{2} \mathrm{O}$ ) with the internal standard agomelatine (an antidepressant drug). Subsequently, $300 \mu \mathrm{l}$ of methanol was added to $100 \mu \mathrm{l}$ of the resulting mixture, followed by centrifugation at $15,000 \mathrm{~g}$ for $20 \mathrm{~min}$. The supernatant was transferred to a new Eppendorf vial for a second centrifugation (15,000 $\mathrm{g}$ for $20 \mathrm{~min}$ ). The final concentration of agomelatine is $2 \mu \mathrm{M}$. Each supernatant was transferred to an auto sampler vial for analysis (see below)

For atazanavir metabolism in liver, liver samples were harvested $30 \mathrm{~min}$ after the treatment of atazanavir (i.v., $30 \mathrm{mg} / \mathrm{kg}$ ). Briefly, livers were weighted and homogenized in water $/ \mathrm{MeOH}$ with the internal standard agomelatine (100 mg liver in $300 \mu \mathrm{l}$ of $\mathrm{H}_{2} \mathrm{O} / \mathrm{MeOH}(\mathrm{v} / \mathrm{v} 3: 1)$ ). Subsequently, $300 \mu \mathrm{l}$ of methanol was added to $100 \mu \mathrm{l}$ of the resulting mixture, followed by centrifugation at $15,000 \mathrm{~g}$ for $20 \mathrm{~min}$. The supernatant was transferred to a new Eppendorf vial for a second centrifugation $(15,000 \mathrm{~g}$ for $20 \mathrm{~min})$. The final concentration of agomelatine is $2 \mu \mathrm{M}$ in samples. Each supernatant was transferred to an auto sampler vial. Five microliters of each prepared sample was injected to a system combining ultra-high performance liquid chromatography (UHPLC) and quadruple time-of-flight mass spectrometry (QTOFMS) for analysis.

Mass spectrometry (UHPLC-QTOFMS analysis). Metabolites from gefitinib and atazanavir were separated using a 1260 Infinity Binary LC System (Agilent Technologies, Santa Clara, CA) equipped with $100 \times 2.1 \mathrm{~mm}$ (Agilent XDB C18) column. The column temperature was maintained at $40{ }^{\circ} \mathrm{C}$. The flow rate of was 0.3 $\mathrm{ml} / \mathrm{min}$, with a gradient ranging from 2 to $98 \%$ aqueous acetonitrile containing $0.1 \%$ formic acid in a 15 -min run. QTOFMS was operated in positive mode with electrospray ionization. Ultra highly pure nitrogen was applied as the drying gas (12 $1 / \mathrm{min}$ ) and the collision gas. The drying gas temperature was set at $325^{\circ} \mathrm{C}$ and nebulizer pressure was kept at 35 psi. The capillary voltages were set at $3.5 \mathrm{kV}$. During mass spectrometry, real-time mass correction and accurate mass were achieved by continuously measuring standard reference ions at $\mathrm{m} / z 121.0508$, 922.0098 in the positive mode. Mass chromatograms and mass spectra were acquired by MassHunter Workstation data Acquisition software (Agilent, Santa Clara, CA) in centroid and profile formats from $\mathrm{m} / z 50$ to 1000 . The acquisition rate was set as 1.5 spectra per $\mathrm{s}$. The method used in this study has been validated by our previous study of gefitinib metabolism in human liver microsomes ${ }^{39}$. Meanwhile, the quality control samples were performed every 10 samples in the process of the sample running. Due to the authentic compounds of metabolites not available, the metabolite identification was based on their exact mass and MS/MS fragments. The chromatograms and relative abundance of metabolite were performed on Qualitative Analysis software (Agilent, Santa Clara, CA). The relative abundance was evaluated based on integrated peak area of each metabolite.

Statistics. Sample sizes for experiments were determined by estimated differences between groups and availability of highly humanized mice. No randomization of animals before allocation to experimental groups nor blinding of experimental groups was done. Statistical analysis was performed using PRISM version $6.0 \mathrm{soft}-$ ware (Graph Pad software) using Mann-Whitney test or ANOVA. Statistical significance was assumed with a $P$ value $<0.05\left(^{*}\right)$. Bars in graphs represent mean $\pm \mathrm{s}$. e.m. unless noted otherwise. Group size $(N)$ represents biological sample size.

Data availability. All data generated or analyzed during this study are included in this published article (and its supplementary information files) with the exception of RNA Seq data. The RNA-Seq data is available from European Nucleotide Archive, ENA accession code PRJEB14714.

Received: 5 October 2016 Accepted: 2 May 2017

Published online: 28 June 2017

\section{References}

1. Olson, H. et al. Concordance of the toxicity of pharmaceuticals in humans and in animals. Regul. Toxicol. Pharmacol. 32, 56-67 (2000).

2. Nelson, D. R. et al. Comparison of cytochrome P450 (CYP) genes from the mouse and human genomes, including nomenclature recommendations for genes, pseudogenes and alternative-splice variants. Pharmacogenetics 14, 1-18 (2004).

3. Guengerich, F. P. \& Cheng, Q. Orphans in the human cytochrome P450 superfamily: approaches to discovering functions and relevance in pharmacology. Pharmacol. Rev. 63, 684-699 (2011).

4. Mercer, D. F. et al. Hepatitis C virus replication in mice with chimeric human livers. Nat. Med. 7, 927-933 (2001).

5. Bissig, K. D., Le, T. T., Woods, N. B. \& Verma, I. M. Repopulation of adult and neonatal mice with human hepatocytes: a chimeric animal model. Proc. Natl Acad. Sci. USA 104, 20507-20511 (2007).

6. Dandri, M. et al. Repopulation of mouse liver with human hepatocytes and in vivo infection with hepatitis B virus. Hepatology 33, 981-988 (2001).

7. Azuma, H. et al. Robust expansion of human hepatocytes in $\mathrm{Fah}^{-/-} / \mathrm{Rag}^{-/-}$ Il2rg- $\mathrm{rg}^{-}$mice. Nat. Biotechnol. 25, 903-910 (2007).

8. Hasegawa, M. et al. The reconstituted 'humanized liver' in TK-NOG mice is mature and functional. Biochem. Biophys. Res. Commun. 405, 405-410 (2011)

9. Washburn, M. L. et al. A humanized mouse model to study hepatitis C virus infection, immune response, and liver disease. Gastroenterology 140, 1334-1344 (2011).

10. Suemizu, H. et al. Establishment of a humanized model of liver using NOD/ Shi-scid IL2Rg null mice. Biochem. Biophys. Res. Commun. 377, 248-252 (2008)

11. Heckel, J. L., Sandgren, E. P., Degen, J. L., Palmiter, R. D. \& Brinster, R. L. Neonatal bleeding in transgenic mice expressing urokinase-type plasminogen activator. Cell 62, 447-456 (1990).

12. Tateno, C. et al. Near completely humanized liver in mice shows human-type metabolic responses to drugs. Am. J. Pathol. 165, 901-912 (2004).

13. Samuelsson, K. et al. Troglitazone metabolism and transporter effects in chimeric mice: a comparison between chimeric humanized and chimeric murinized FRG mice. Xenobiotica 44, 186-195 (2014).

14. Lootens, L. et al. Steroid metabolism in chimeric mice with humanized liver. Drug Test Anal. 1, 531-537 (2009).

15. Foster, J. R. et al. Differential effect of troglitazone on the human bile acid transporters, MRP2 and BSEP, in the PXB hepatic chimeric mouse. Toxicol. Pathol. 40, 1106-1116 (2012).

16. Xu, D. et al. Fialuridine induces acute liver failure in chimeric TK-NOG mice: a model for detecting hepatic drug toxicity prior to human testing. PLoS Med. 11, e1001628 (2014) 
17. Bateman, T. J., Reddy, V. G., Kakuni, M., Morikawa, Y. \& Kumar, S Application of chimeric mice with humanized liver for study of human-specific drug metabolism. Drug Metab. Dispos. 42, 1055-1065 (2014).

18. Nishimura, T. et al. Using chimeric mice with humanized livers to predict human drug metabolism and a drug-drug interaction. J. Pharmacol. Exp. Ther. 344, 388-396 (2013).

19. Tanoue, C. et al. Prediction of human metabolism of the sedative-hypnotic zaleplon using chimeric mice transplanted with human hepatocytes. Xenobiotica 43, 956-962 (2013).

20. Bissig, K. D. et al. Human liver chimeric mice provide a model for hepatitis B and C virus infection and treatment. J. Clin. Invest. 120, 924-930 (2010).

21. Meuleman, P. et al. Morphological and biochemical characterization of a human liver in a uPA-SCID mouse chimera. Hepatology 41, 847-856 (2005)

22. Kato, K. et al. Development of murine Cyp3a knockout chimeric mice with humanized liver. Drug Metab. Dispos. 43, 1208-1217 (2015).

23. Nakada N. et al. Murine Cyp3a knockout chimeric mice with humanized liver: prediction of the metabolic profile of nefazodone in humans. Biopharm. Drug Dispos. 37, 3-14 (2016).

24. Shen, A. L., O’Leary, K. A. \& Kasper, C. B. Association of multiple developmental defects and embryonic lethality with loss of microsomal NADPH-cytochrome P450 oxidoreductase. J. Biol. Chem. 277, 6536-6541 (2002).

25. Wu, L. et al. Conditional knockout of the mouse NADPH-cytochrome p450 reductase gene. Genesis 36, 177-181 (2003).

26. Henderson, C. J. et al. Inactivation of the hepatic cytochrome P450 system by conditional deletion of hepatic cytochrome P450 reductase. J. Biol. Chem. 278, 13480-13486 (2003).

27. Gu, J. et al. Liver-specific deletion of the NADPH-cytochrome P450 reductase gene: impact on plasma cholesterol homeostasis and the function and regulation of microsomal cytochrome $\mathrm{P} 450$ and heme oxygenase. J. Biol. Chem. 278, 25895-25901 (2003).

28. Thomas, K. R. \& Capecchi, M. R. Site-directed mutagenesis by gene targeting in mouse embryo-derived stem cells. Cell 51, 503-512 (1987).

29. Skarnes, W. C. et al. A conditional knockout resource for the genome-wide study of mouse gene function. Nature 474, 337-342 (2011).

30. Farley, F. W., Soriano, P., Steffen, L. S. \& Dymecki, S. M. Widespread recombinase expression using FLPeR (flipper) mice. Genesis 28, 106-110 (2000).

31. Haft, D. H., Selengut, J., Mongodin, E. F. \& Nelson, K. E. A guild of 45 CRISPRassociated (Cas) protein families and multiple CRISPR/Cas subtypes exist in prokaryotic genomes. PLoS Comput. Biol. 1, e60 (2005).

32. Jinek, M. et al. A programmable dual-RNA-guided DNA endonuclease in adaptive bacterial immunity. Science 337, 816-821 (2012).

33. Jansen, R., Embden, J. D., Gaastra, W. \& Schouls, L. M. Identification of genes that are associated with DNA repeats in prokaryotes. Mol. Microbiol. 43 1565-1575 (2002).

34. Bissig-Choisat, B. et al. Development and rescue of human familial hypercholesterolaemia in a xenograft mouse model. Nat. Commun. 6, 7339 (2015).

35. Weng, Y., DiRusso, C. C., Reilly, A. A., Black, P. N. \& Ding, X. Hepatic gene expression changes in mouse models with liver-specific deletion or global suppression of the NADPH-cytochrome P450 reductase gene. Mechanistic implications for the regulation of microsomal cytochrome P450 and the fatty liver phenotype. J. Biol. Chem. 280, 31686-31698 (2005).

36. Williams, J. A. et al. Drug-drug interactions for UDP-glucuronosyltransferase substrates: a pharmacokinetic explanation for typically observed low exposure (AUCi/AUC) ratios. Drug Metab. Dispos. 32, 1201-1208 (2004).

37. Barker, A. J. et al. Studies leading to the identification of ZD1839 (IRESSA): an orally active, selective epidermal growth factor receptor tyrosine kinase inhibitor targeted to the treatment of cancer. Bioorg. Med. Chem. Lett. 11, 1911-1914 (2001).

38. Herbst, R. S., Fukuoka, M. \& Baselga, J. Gefitinib--a novel targeted approach to treating cancer. Nat. Rev. Cancer 4, 956-965 (2004).

39. Liu, X. et al. Metabolomics reveals the formation of aldehydes and iminium in gefitinib metabolism. Biochem. Pharmacol. 97, 111-121 (2015).

40. McKillop, D. et al. Metabolic disposition of gefitinib, an epidermal growth factor receptor tyrosine kinase inhibitor, in rat, dog and man. Xenobiotica 34, 917-934 (2004)

41. Scheffler, M., Di Gion, P., Doroshyenko, O., Wolf, J. \& Fuhr, U. Clinical pharmacokinetics of tyrosine kinase inhibitors: focus on 4-anilinoquinazolines. Clin. Pharmacokinet. 50, 371-403 (2011).

42. Li, F., Lu, J., Wang, L. \& Ma, X. CYP3A-mediated generation of aldehyde and hydrazine in atazanavir metabolism. Drug Metab. Dispos. 39, 394-401 (2011).

43. Baillie, T. A. Future of toxicology-metabolic activation and drug design: challenges and opportunities in chemical toxicology. Chem. Res. Toxicol. 19, 889-893 (2006).

44. Guengerich, F. P. \& MacDonald, J. S. Applying mechanisms of chemical toxicity to predict drug safety. Chem. Res. Toxicol. 20, 344-369 (2007).
45. Dalvie, D. et al. Assessment of three human in vitro systems in the generation of major human excretory and circulating metabolites. Chem. Res. Toxicol. 22, 357-368 (2009)

46. Anderson, S., Luffer-Atlas, D. \& Knadler, M. P. Predicting circulating human metabolites: how good are we ? Chem. Res. Toxicol. 22, 243-256 (2009).

47. Pettitt, S. J. et al. Agouti C57BL/6N embryonic stem cells for mouse genetic resources. Nat. Methods 6, 493-495 (2009).

48. Cradick, T. J., Qiu, P., Lee, C. M., Fine, E. J. \& Bao, G. COSMID: a web-based tool for identifying and validating CRISPR/Cas off-target sites. Mol. Ther. Nucleic Acids 3, e214 (2014).

49. Hwang, W. Y. et al. Efficient genome editing in zebrafish using a CRISPR-Cas system. Nat. Biotechnol. 31, 227-229 (2013).

50. Cong, L. et al. Multiplex genome engineering using CRISPR/Cas systems. Science 339, 819-823 (2013).

51. Ponder, K. P. et al. Mouse hepatocytes migrate to liver parenchyma and function indefinitely after intrasplenic transplantation. Proc. Natl Acad. Sci. USA 88, 1217-1221 (1991)

52. Li, B. \& Dewey, C. N. RSEM: accurate transcript quantification from RNA-Sec data with or without a reference genome. BMC. Bioinformatics 12, 323 (2011).

53. Langmead, B. \& Salzberg, S. L. Fast gapped-read alignment with Bowtie 2. Nat. Methods 9, 357-359 (2012).

54 Eisenberg, E. \& Levanon, E. Y. Human housekeeping genes, revisited. Trends Genet. 29, 569-574 (2013).

\section{Acknowledgements}

We thank Nathan Nowak, Stephanie Sandor, Isabel Lorenzo, Jason Heaney, Rui Chen and Jeffrey Howard, Robert Kruse and Angie Cohns for technical assistance, V.L. Brandt and C. Gillespie for critical comments on the manuscript, and Hoang Nguyen for sharing reagents. K.D.B. is supported by the National Heart Lung and Blood Institute (NHLBI) grant R01HL134510, the Texas Hepatocellular Carcinoma Consortium (THCCC) (CPRIT \#RP150587) and the Diana Helis Henry and Adrienne Helis Malvin Medical Research Foundations. F.P.P. and B.B.C. were supported by T32HL092332. The DLDCC is supported by P30CA125123. CMM core facility of Texas Medical Center Digestive Disease Center (P30-DK56338). NCI Cancer Center Support Grant (P30CA125123) for the Mouse Embryonic Stem Cell Core and the Genetically Engineered Mouse Core at Baylor College of Medicine.

\section{Author contributions}

K.D.B. and M.B.: Designed the experiments. M.B. and B.B.C.: Performed in vivo experiments. M.B.: Did immunostaining and Southern blot. D.Y.: Prepared libraries and run sequencing. X. L.: Did western blotting and PCRs. M.B. and X.L.: Did qPCRs. M.B., F.P.P., and K.D.B.: Designed and prepared CRISPR/Cas9 knock-out approach. X. Li. and F.L.: Performed analysis of drug metabolites. B.Z. and P.S.: Did bioinformatics. All authors read and approved the final manuscript.

\section{Additional information}

Supplementary Information accompanies this paper at doi:10.1038/s41467-017-00049-x.

Competing interests: The authors declare no competing financial interests.

Reprints and permission information is available online at http://npg.nature.com/ reprintsandpermissions/

Publisher's note: Springer Nature remains neutral with regard to jurisdictional claims in published maps and institutional affiliations.

Change History: An incorrect version of the Supplementary Information was inadvertently published with this Article where the wrong file was included. The HTML has been updated to include the correct version of the Supplementary Information.

Open Access This article is licensed under a Creative Commons Attribution 4.0 International License, which permits use, sharing, adaptation, distribution and reproduction in any medium or format, as long as you give appropriate credit to the original author(s) and the source, provide a link to the Creative Commons license, and indicate if changes were made. The images or other third party material in this article are included in the article's Creative Commons license, unless indicated otherwise in a credit line to the material. If material is not included in the article's Creative Commons license and your intended use is not permitted by statutory regulation or exceeds the permitted use, you will need to obtain permission directly from the copyright holder. To view a copy of this license, visit http://creativecommons.org/ licenses/by/4.0/.

(C) The Author(s) 2017 